\title{
Porfiria cutánea tarda. Caso clínico
}

\author{
NICOLÁS HERMOSILLA B. ${ }^{1}$, GONZALO DE TORO², \\ MONTSERRAT MOLGÓ1,3
}

\section{Porphyria cutanea tarda. Case report}

Porphyria cutanea tarda (PCT) is the most common type of porphyria: it is characterized by blistering lesions, erosions and crusts on the back of the hands, associated with photosensitivity and facial hypertrichosis. It is produced by acquired or hereditary deficiency of the enzyme UROD, fifth enzyme in the chain of production of the Heme group. This causes accumulation of porphyrins in the liver, which are subsequently mobilized to the skin, where lesions are generated by photosensitivity. This deficiency can be exacerbated by multiple causes. We report a 51-year-old female presenting with the characteristic dermal lesions described above, which disappeared when she discontinued her hormone replacement therapy with estradiol and dydrogesterone. Urinary and blood uroporphyrin and hexacarboxyl porphyrins were elevated and plasma ferritin was $479 \mathrm{ng} /$ $\mathrm{ml}$. Hormone replacement therapy was discontinued and phlebotomies were attempted but not tolerated by the patient. The dermic lesions have not relapsed.

(Rev Med Chile 2018; 146: 943-946)

Key words: Estrogens; Porphyrias; Porphyria Cutanea Tarda.
${ }^{1}$ Escuela de Medicina, Facultad de Medicina, Pontificia Universidad Católica de Chile. Santiago, Chile. ¿2Servicio de Anatomía Patológica, Hospital Puerto Montt

Dr. Eduardo Schütz Schroeder. Puerto Montt, Chile.

${ }^{3}$ Departamento de Dermatología. Escuela de Medicina. Facultad de Medicina, Pontificia Universidad Católica de Chile. Santiago, Chile.

Los autores declaran no tener conflictos de interés.

Trabajo no recibió

financiamiento.

Recibido el 21 de diciembre de 2017, aceptado el 20 de agosto de 2018.

Correspondencia a: Nicolás Hermosilla Bravo Noruega 6595 - 1904. nehermosilla@uc.cl
I as porfirias son un grupo de enfermedades raras caracterizadas por la deficiencia de alguna enzima de la cadena de producción del grupo heme. Existen diferentes formas de porfiria, dependiendo de qué enzima esté afectada ${ }^{1}$, pero todas culminan en la acumulación de precursores de porfirinas o porfirinas en el hígado o médula ósea. El órgano donde se acumulan preferentemente estas se utiliza para clasificar los tipos de porfirias y predecir los signos y síntomas de presentación. En el caso de la porfiria cutánea tarda (PCT), la más frecuente de las porfirias, la enzima afectada es la uroporfirinógeno decarboxilasa (UROD) y la acumulación ocurre en el hígado².

\section{Caso clínico}

Se presenta una paciente de 51 años sin antecedentes médicos de importancia, usuaria de terapia de reemplazo hormonal (TRH) (estradiol $1 \mathrm{mg}+$ didrogesterona $5 \mathrm{mg}$ ) y previamente anticonceptivos orales combinados por 24 años (etinilestradiol $0,02 \mathrm{mg}+$ drospirenona $3 \mathrm{mg}$ ), sin hábito tabáquico ni consumo de alcohol. Consultó por lesiones en dorso de las manos con difícil cicatrización de 5 meses de evolución. A la anamnesis dirigida no refería episodios previos. Al examen físico se evidenciaban pequeñas erosiones, costras y pequeñas ampollas tensas en el dorso de las manos (Figuras 1 y 2). Además, presentaba hipertricosis periorbitaria. Destaca que durante 1 mes de suspensión de TRH, por realización de exámenes hormonales, las lesiones desaparecieron hasta reinicio de la TRH. En exámenes de laboratorio destacaba: determinación de porfirinas en orina $24 \mathrm{~h}$ (Laboratorio UC); uroporfirinas: $1.086 \mathrm{mcg} / \mathrm{L}$ (valor de referencia (VR) $<21 \mathrm{mc}$ $\mathrm{g} / \mathrm{L})$ y heptacarboxiporfirina: $826 \mathrm{mcg} / \mathrm{L}(\mathrm{VR}<3$ $\mathrm{mcg} / \mathrm{L})$; porfirinas en plasma con método de cromatografía en capa fina y espectrofotometría UV (Clínica Mayo, Rochester, Minnesota, EE.UU.): uroporfirinas $4,4 \mathrm{mcg} / \mathrm{dL}(\mathrm{VR} \leq 0,1 \mathrm{mcg} / \mathrm{dL})$, heptacarboxiporfirina: $3,3 \mathrm{mcg} / \mathrm{dL}(\mathrm{VR} \leq 0,1 \mathrm{mcg} /$ $\mathrm{dL})$ y hexacarboxiporfirina $1,6 \mathrm{mcg} / \mathrm{dL}(\mathrm{VR} \leq 0,1$ 


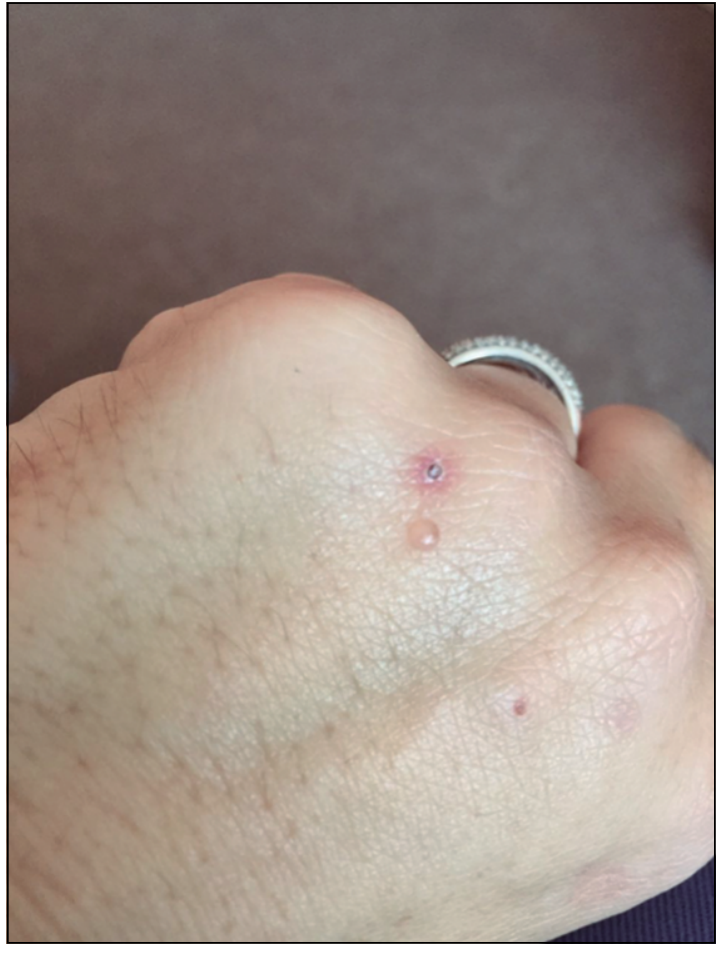

Figura 1. Lesión ampollar en dorso de mano izquierda. $\mathrm{mcg} / \mathrm{dL}$ ), patrón compatible con PCT, ferritina de 479 y perfil hepático con leve elevación de las transaminasas (SGOT 33 y SGPT 63), sin otras alteraciones. Además, se realizó biopsia de una lesión no ampollar, ya que no existían al momento de la biopsia. Esta fue informada como: piel con úlcera cutánea, marcado edema dérmico e infiltrado inflamatorio linfocitario y polimorfonuclear neutrófilo, siendo inespecífica.

Una vez hecho el diagnóstico de PCT, se suspendió inmediatamente la TRH y se inició tratamiento con flebotomías de $450 \mathrm{ml}$ cada 10 días, con mala tolerancia a la segunda sesión, posterior a esta, se evidenció un hematocrito de control de $33 \%$, por lo que se distanció la siguiente flebotomía 1 mes y se disminuyó la cantidad de sangre extraída a $300 \mathrm{ml}$. Luego de 3 sesiones de flebotomía fue evaluada por hematología, suspendiendo tratamiento, debido a que se observara desaparición de manifestaciones clínicas y normalización de pruebas hepáticas y ferritina.

Posterior a la suspensión de TRH no ha vuelto a presentar lesiones, solo persiste con hipertricosis facial en tratamiento con terapia láser.

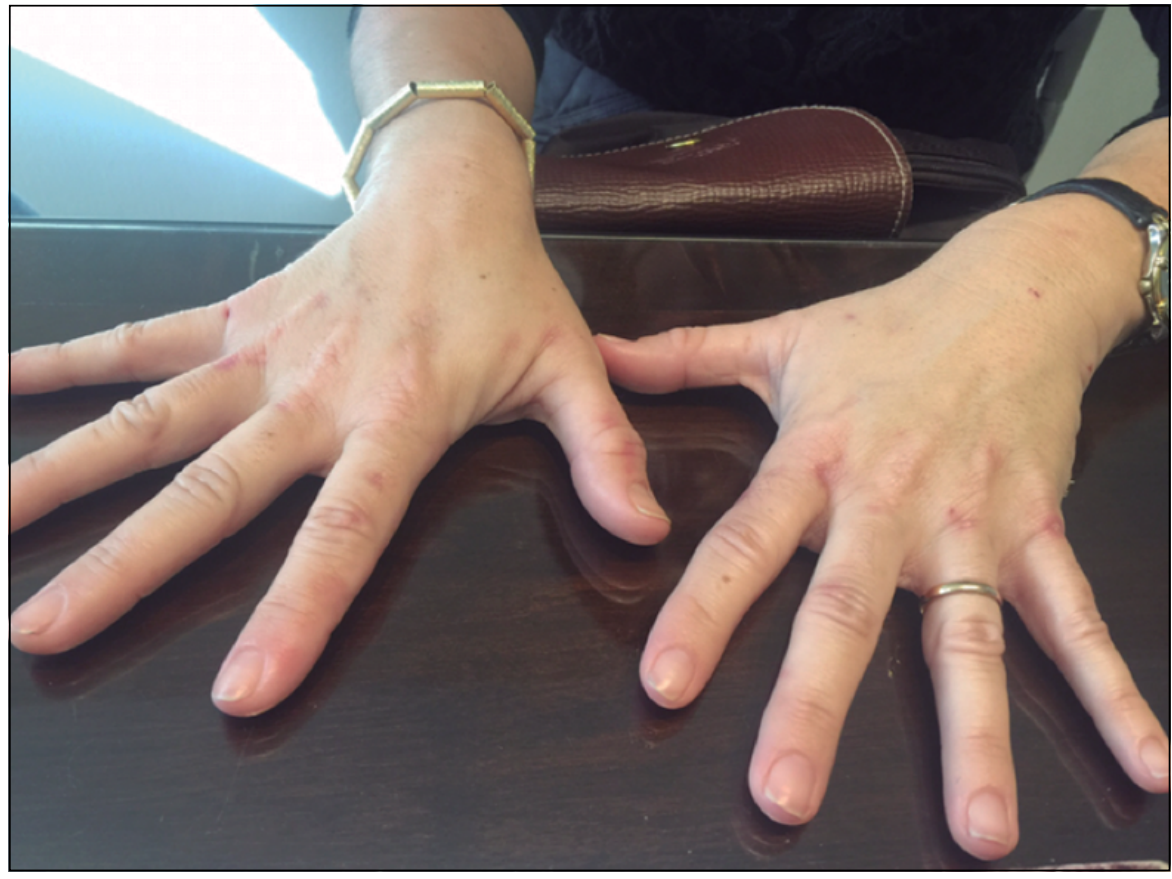

Figura 2. Lesiones cicatriciales en dorso de manos. 


\section{Discusión}

La PCT es la más común de las porfirias, con una prevalencia global de uno por cada 10.000 personas, aunque esto varía según zona geográfica. No existe diferencias en la prevalencia por sexo ${ }^{3}$. Las manifestaciones clínicas aparecen en la edad media de la vida, generalmente asociado a la exposición a algún factor desencadenante.

\section{Se describen 3 formas de presentación:}

- Tipo I: Es la forma adquirida o esporádica, abarcando 70-80\% de los casos de PCT. No hay una mutación genética que la produzca, el déficit enzimático es consecuencia de una infección viral o sustancias exógenas, se encuentra solo en el hígado y se presenta generalmente en personas de 40-60 años, asociado a factores precipitantes ${ }^{4}$.

- Tipo II: Forma familiar o hereditaria, responsable de $20 \%$ de los casos $^{2}$. Se hereda en forma autosómica dominante y la actividad enzimática está reducida aproximadamente $50 \%$ en todos los tejidos. La expresión clínica de la enfermedad depende de la predisposición genética y de factores desencadenantes ${ }^{4}$.

- Tipo III: Resulta de una mutación heredada de los familiares de primer grado con PCT tipo II confirmado. La población diagnosticada con PCT tipo III tiene actividad normal de UROD eritrocítica, pero igualmente desarrollan manifestaciones clásicas de $\mathrm{PCT}^{5}$.

Clínicamente, la PCT se caracteriza por presentar fotosensibilidad en áreas fotoexpuestas y aumento de la fragilidad cutánea. Además, se pueden observar quistes de milluim, vesículas, bulas, erosiones y costras, las cuales se ubican preferentemente en el dorso de las manos, pero también se pueden encontrar en antebrazo, cara, piernas y pies. Estas lesiones pueden dejar cicatriz y atrofia de la piel. Signos como la hipertricosis periorbitaria y prurito son más frecuentes en mujeres.

Para formular el diagnóstico de PCT la clínica es insuficiente, por lo que se debe realizar medición de porfirinas en sangre, orina y heces. Ya que las porfirinas estarán elevadas en todas las porfirias, lo que permitirá el diagnóstico exacto es la evaluación del precursor del grupo heme que está elevado. En el caso de PCT hay una pro- porción aumentada de uroporfirina I respecto a uroporfirina III $^{7}$.

El estudio histopatológico compatible con PCT no es requisito para el diagnóstico. Si la biopsia es tomada de una ampolla, se evidencia un estrato córneo compacto, con epitelio necrótico, escaso infiltrado linfocítico y elastosis actínica en la porción superior. Los "cuerpos oruga", si bien son característicos de la PCT, observándose como glóbulos eosinofílicos lineales, PAS positivos, en el espesor de la epidermis suprayacente a la zona de desprendimiento subepidérmico, también pueden observarse en pseudoporfiria y protoporfiria eritropoyética. Adicionalmente, se observan papilas dérmicas onduladas o en "base festoneada", con infiltrado inflamatorio escaso en dermis superior y depósito de material hialino PAS positivo confinado a la pared de los vasos dérmicos y en el tejido conectivo perivascular inmediato, lo cual se observa más frecuentemente en las lesiones crónicas ${ }^{8}$. Los hallazgos de la inmunofluorescencia directa demuestran depósitos de IgG y, menos comúnmente, de IgM y C3 en los vasos de la dermis superficial. La microscopía electrónica muestra una marcada reduplicación de la lámina basal de los vasos y, ocasionalmente, reduplicación de la membrana basal de la unión dermoepidérmica.

Los pacientes con PCT tipo II tienen predisposición genética a una menor actividad de la uroporfirinógeno decarboxilasa (UROD), quinta enzima de la vía metabólica del grupo heme, encargada del paso de uroporfirinógeno III a coproporfirinógeno III. Esta disfunción se intensifica por distintas noxas, y cuando la actividad de la UROD es menor a $20 \%$ se acumulan porfirinas en el hígado y luego se transportan a la piel, donde son fotoactivadas, siendo las responsables de la expresión clínica de esta enfermedad. Esto explica la aparición tardía, a pesar de que la que la alteración genética siempre estuvo presente ${ }^{8}$.

Se han descrito múltiples factores desencadenantes (noxas) de PCT, entre ellos el alcohol, tabaco, uso de estrógenos exógenos, olmesartan, asociación con virus hepatitis C (VHC) y hemocromatosis. Se cree que la mayoría de estos factores alteran la función hepática de la UROD a través de un mecanismo dependiente del hierro, lo que explica la presencia de parámetros de sobrecarga de hierro en pacientes con PCT ${ }^{9}$.

Está bien documentada la relación entre PCT y uso de estrógenos exógenos, sin conocerse el 
mecanismo exacto. Se sabe que el dietilestilbestrol induce la ALA sintasa hepática, pero no está claro cómo esto lleva a la expresión o exacerbación de la enfermedad ${ }^{10}$.

El tratamiento para los diferentes tipos de PCT es el mismo. Consiste en realizar flebotomías periódicas, con el fin de disminuir el hierro al limite inferior normal. Se recomienda extraer $450 \mathrm{ml}$ de sangre cada 2 semanas, con control de hemoglobina, la cual no debe caer bajo $10 \mathrm{~g} / \mathrm{dL}$. En pacientes con enfermedad cardiovascular o pulmonar, en que la flebotomía este contraindicada, se puede iniciar tratamiento con antimaláricos, como cloroquina o hidroxicloroquina.

Singal y cols ${ }^{11}$ evaluaron el uso de flebotomías cada 2 semanas vs hidroxicloroquina $100 \mathrm{mg} 2$ veces por semana. Se enrolaron 30 pacientes con PCT bien documentada, a 17 pacientes se les realizó flebotomía y 13 recibieron hidroxicloroquina. La media de remisión bioquímica de la enfermedad, definida como normalización de los niveles urinarios de porfirinas, fue en ambos grupos entre 6-7 meses y la remisión clínica entre 2-3 meses, sin diferencias estadísticamente significativas.

\section{Conclusión}

Se presentó el caso de una paciente con diagnóstico de PCT, probablemente inducido por estrógenos exógenos, en que la suspensión de la terapia de reemplazo hormonal detuvo la aparición de lesiones. Si bien esta relación está descrita, hacen falta más estudios para comprender el mecanismo por el cual esto ocurre.

\section{Referencias}

1. Balwani M, Desnick RJ. The porphyrias: advances in diagnosis and treatment. Hematol Am Soc Hematol Educ Program 2012; 2012: 19-27.
2. Handler NS, Handler MZ, Stephany MP, Handler GA, Schwartz RA. Porphyria cutanea tarda: an intriguing genetic disease and marker. Int J Dermatol 2017; 56 (6): e106-17.

3. Elder GH. The cutaneous porphyrias. Semin Dermatol. 1990; 9 (1): 63-9.

4. Montardit AL, Carmona LE, Capelli L, Sanz A, Oborski MR. Porfiria cutánea tarda; Comunicación de un caso, Arch. Argent. Dermatol 2013; 63 (5): 200-3.

5. Lambrecht RW, Thapar M, Bonkovsky HL. Genetic aspects of porphyria cutanea tarda. Semin Liver Dis 2007; 27 (1): 99-108.

6. Muñoz-Santos C, Guilabert A, Moreno N, To-Figueras J, Badenas C, Darwich E, et al. Familial and sporadic porphyria cutanea tarda: clinical and biochemical features and risk factors in 152 patients. Medicine (Baltimore) 2010; 89 (2): 69-74.

7. Horner ME, Alikhan A, Tintle S, Tortorelli S, Davis DMR, Hand JL. Cutaneous porphyrias part I: epidemiology, pathogenesis, presentation, diagnosis, and histopathology. Int J Dermatol 2013; 52 (12): 146480.

8. Sánchez CMF, Navarrete CJ, Buchroithner HC. Porfiria cutánea tarda: Revisión de la literatura a propósito de un caso. Rev Chil Dermatol 2015; 31 (3): 265-71.

9. Muñoz-Santos C, Guilabert A, Moreno N, Giménez M, Darwich E, To-Figueras J, et al. The association between porphyria cutanea tarda and diabetes mellitus: analysis of a long-term follow-up cohort. Br J Dermatol 2011; 165 (3): 486-91.

10. Cruz-Rojo J, Fontanellas A, Morán-Jiménez MJ, Navarro-Ordóñez S, García-Bravo $M$, Méndez $M$, et al. Precipitating/aggravating factors of porphyria cutanea tarda in Spanish patients. Cell Mol Biol Noisy-Gd Fr 2002; 48 (8): 845-52.

11. Singal AK, Kormos-Hallberg C, Lee C, Sadagoparamanujam V-M, Grady JJ, Freeman DH, et al. Low-Dose Hydroxychloroquine is as Effective as Phlebotomy in Treatment of Patients with Porphyria Cutanea Tarda. Clin Gastroenterol Hepatol Off Clin Pract J Am Gastroenterol Assoc 2012; 10 (12): 1402-9. 